\title{
3 Research Square

\section{The structure-activity relationship between precursor fine structure and cathodes performance in Ni-rich layered oxide}

Lang Qiu

Sichuan University

Mengke Zhang

Sichuan University

Kanghui Hu

Sichuan University

Yang Song

Sichuan University

Zhenguo Wu

Sichuan University

Luchao Yue

University of Electronic Science and Technology of China

Wei Xiang

Sichuan University

Yong Ming

Sungkyunkwan University (SKKU)

Gong-Ke Wang

Henan Normal University

Yu-Xia Liu

Shaanxi University of Science and Technology https://orcid.org/0000-0003-1139-8563

Yan Sun

13207516703

Jun Zhang

Sichuan University

Bin Zhang

Yibin Libao New Material Co., Ltd

Xiaodong Guo ( $\nabla$ xiaodong2009@163.com )

Sichuan University https://orcid.org/0000-0003-2180-1985 
Keywords: lithium ion batteries, primary particle size, precursor growth mechanism

Posted Date: November 15th, 2021

DOI: https://doi.org/10.21203/rs.3.rs-1046227/v1

License: (c) (1) This work is licensed under a Creative Commons Attribution 4.0 International License. Read Full License 


\section{The structure-activity relationship between precursor fine structure and cathodes performance in Ni-rich layered oxide}

Lang Qiu ${ }^{1}$, Mengke Zhang ${ }^{1}$, Kanghui $\mathrm{Hu}^{1}$, Yang Song ${ }^{1 *}$, Zhenguo $\mathrm{Wu}^{1}$, Luchao Yue ${ }^{1,2}$,

Wei Xiang ${ }^{3}$, Yong Ming ${ }^{4}$, Gongke Wang ${ }^{5}$, Yuxia Liu ${ }^{6}$, Yan Sun ${ }^{7}$, Jun Zhang ${ }^{1,8}$, Bin Zhang $^{9}$, Xiaodong Guo ${ }^{1 *}$

${ }^{1}$ College of Chemical Engineering, Sichuan University, Chengdu, 610065, PR China.

${ }^{2}$ University of Electronic Science and Technology of China.

${ }^{3}$ College of Materials and Chemistry \& Chemical Engineering, Chengdu University of Technology, Chengdu, 610059, PR China.

${ }^{4}$ School of Chemical Engineering, Sungkyunkwan University (SKKU), Suwon 16419 Republic of Korea.

${ }^{5}$ School of Materials Science and Engineering, Henan Normal University.

${ }^{6}$ The Key Laboratory of Life-Organic Analysis, Key Laboratory of Pharmaceutical, Intermediates and Analysis of Natural Medicine, School of Chemistry and Chemical Engineering, Qufu Normal University, Qufu, 273165, Shandong, China.

${ }^{7}$ School of Mechanical Engineering, Chengdu University, Chengdu, 610106, China.

${ }^{8}$ Ruyuan Dongyang Guangcixing Material Limited Company, Shaoguan, Guangdong 512710,PR China.

${ }^{9}$ Yibin Libao New Material Co., Ltd, Yibin, China.

* Corresponding authors:

*E-mail addresses:

*E-mail addresses: xiaodong2009@scu.edu.cn 
Cathode's primary particle structure plays a key role in the performance of lithium ion batteries, which can be controlled by the precursor synthesis. Regretfully, the relevance between primary particle structure and cathode performance is not explicitly elucidated, that is, what is the discrepancy of cathode's primary particle size on the structural degradation? In order to elaborate the structure-activity relationship between them, we have systematically investigated the regulation of primary particle size through an in-depth analysis of the precursor growth mechanism, ammonia-stirring coupling and hydrodynamics optimization. Structural and electrochemical characterizations of $\mathrm{LiNi}_{0.92} \mathrm{Co}_{0.04} \mathrm{Mnn}_{0.04} \mathrm{O}_{2}$ with different primary sizes $(336,447,565$ and $675 \mathrm{~nm})$ and a rounded analysis of structural degradation after cycling provide insight into the correlation between precursor fine structure and cathode performance, i.e. larger cathode's primary particle size can effectively inhibit CEI film formation, structure decay, the intragranular/intergranular cracks formation owing to the alleviation of localized stress. 
Ni-rich cathodes have attracted much attention in the field of new energy due to their high capacity and low cost. ${ }^{[1-5]}$ The agglomerated polycrystalline NCM cathodes shorten the diffusion length of primary particles and increases the number of pores, resulting in the accelerated transport of $\mathrm{Li}^{+}$. The traditional $\mathrm{LiNi}_{1-x-y} \mathrm{Co}_{x} \mathrm{Mn}_{y} \mathrm{O}_{2}(\mathrm{NCM})$ cathodes with micro secondary particles assembled by nano primary particles were prepared using coprecipitation method. ${ }^{[6]}$ In the coprecipitation process, there were two main reactions, firstly, metal ions complexed with ammonia, and then, the complex reacted with hydroxyl ion to form precipitation. ${ }^{[7]}$ As far as the precursor growth process is concerned, He et al. reported that the growth rate of (001) crystal plane was the fastest and the $\mathrm{TM}-\mathrm{NH}_{3}$ (TM represents transition metal ions) complex tended to aggregate to (001) crystal plane. Additionally, the whole coprecipitation process included nucleation, growth, agglomeration and re-crystallization. ${ }^{[8]}$ Sun et al demonstrated that the increase of ammonia concentration or the decrease of stirring speed $(\mathrm{pH})$ could increase secondary particle size. Meanwhile, the precursor after high-temperature lithiation could effectively inherit the original morphology and particle size ${ }^{[9,10]}$ Besides, the relationship between ammonia concentration and $\mathrm{pH}$ on the growth of precursors was proposed to predict the optimum conditions for the synthesis of materials $(y=0.7731 \times \ln (x+0.0312)+11.6708, y$ represented $\mathrm{pH}$ value, $x$ represented ammonia concentration). ${ }^{[11]}$ Subsequently, our group also reported the structure evolution of Ni-rich NCA precursors under different ammonia concentration and $\mathrm{pH}$, including the primary particle size increased with the increase of ammonia concentration or the decrease of $\mathrm{pH}^{[12]}$

These studies on the coprecipitation process could be used to regulate precursor structure to ameliorate the inferior cycle stability of agglomerated polycrystalline Ni-rich cathodes because of the (1) intragranular cracks, ${ }^{[13,14]}$ (2) intergranular cracks, ${ }^{[15]}$ (3) layered phase into spinel / rock salt phase deterioration, ${ }^{[16]}$ cathode-electrolyte interface (CEI) film formation, ${ }^{[17]}$ (5) active substances dissolution into electrolyte, ${ }^{[18]}$ (6) electrolyte decomposition. ${ }^{[19]}$ Currently, the precursor structure with radial ordered structure or gradient structure or flower-like accumulation could be synthesized by changing the reaction conditions. ${ }^{[20-25]}$ These 
structures showed that the primary particles had an ordered stacking structure, or the secondary particles had gradient distribution of transition metal ions, which could effectively inhibit the structure degradation and cracks formation. Notably, the conventional precursors without special stacking mode or special distribution of transition metal ions after lithiation reaction also exhibited different cycling stability (with the same element ratio). Combined with these works, we convinced that there was a structure-activity relationship between precursor fine structure and cathode' structure stability.

Herein, we finely regulated the precursors with different primary particle sizes $(182,255,433,520,670 \mathrm{~nm})$ by deeply analyzing the growth mechanism of the precursors and coupling the crystallization process (ammonia concentration, stirring speed) and flow characteristics in the reactor. By coupling ammonia concentration and rotating speed, the precursor with secondary particle size of $\sim 14$ um and primary particle size of $180-550 \mathrm{~nm}$ can be effectively adjusted. By optimizing hydrodynamics to inhibit the secondary nucleation during the precursor growth process, the primary particle size can be adjusted to $\sim 670 \mathrm{~nm}$, but the secondary particle size is $\sim 6$ um (the inhibition of secondary nucleation will lead to the limitation of the subsequent growth of secondary particles). Combined with high temperature lithiation, the differentiation of the cathode's primary particle size on the structural degradation was exposed. The larger cathode' primary particle size could effectively inhibit the CEI film formation, layered phase into spinel / rock salt phase deterioration, the intragranular and intergranular cracks formation. Especially, the cathode with larger primary particle size and the smaller secondary particle size could also effectively enhance cycle stability. Our work indicated that the cathodes could develop towards quasi single crystal in the future.

\section{Results}

\section{Precursor growth mechanism}

The precipitation crystallization reaction process is very complex, which generally involves two processes. Firstly, the precipitation reaction is carried out, secondly, it begins to nucleate and grow when the chemical compound reaches a certain degree of 
supersaturation. For the Ni-Co-Mn $\left(\mathrm{TM}^{2+}: \mathrm{Ni}^{2+}, \mathrm{Co}^{2+}, \mathrm{Mn}^{2+}\right)$ precursor growth process in the system containing ammonia, the following reactions are generally involved (the related synthesis methods and thermodynamic process analysis are exhibited in supporting Methods and section 1): ${ }^{[7]}$

Firstly,

$$
\mathrm{TM}^{2+}+n \mathrm{NH}_{3} \rightleftharpoons\left[\mathrm{TM}\left(\mathrm{NH}_{3}\right)_{n}\right]^{2+}
$$

Secondly,

$$
\left[\mathrm{TM}\left(\mathrm{NH}_{3}\right)_{n}\right]^{2+}+2 \mathrm{OH}^{-} \rightleftharpoons \mathrm{TM}(\mathrm{OH})_{2}+n \mathrm{NH}_{3}
$$

Finally, it begins to nucleate and grow when $\mathrm{TM}(\mathrm{OH})_{2}$ reaches a certain supersaturation. Additionally, dissolution and recrystallization are also involved during the growth process of precursors. In our work, Fig. 1a shows that the crystal structure of $\mathrm{Ni}_{0.92} \mathrm{Co}_{0.04} \mathrm{Mn}_{0.04}(\mathrm{OH})_{2}$ precursor can be indexed as $\beta-\mathrm{Ni}(\mathrm{OH})_{2}$ layered structure $(\mathrm{P} \overline{3} \mathrm{~m} 1)$ for all samples. Compared with other crystal planes in $\beta-\mathrm{Ni}(\mathrm{OH})_{2}$, (101) crystal plane has smaller specific surface energy, which can be retained in the growth process. As shown in Fig. 1a, the relative strength of (101) crystal face increases with the reaction. The D50 size of precursor is relatively stable when the reaction time reaches $50 \mathrm{~h}$ (Fig. 1b, c). Notably, in the whole reaction process, ammonia concentration and solid content increase in the first $32 \mathrm{~h}$, and reach a relatively stable state after $32 \mathrm{~h}$, as shown in Fig. S3. Particularly, the nucleation rate equation is expressed as follows: ${ }^{[26]}$

$$
J=J_{0} \exp \left[-\frac{16 \pi \gamma^{3} v^{2}}{3 k_{\mathrm{B}} R_{\mathrm{g}}^{2} T^{3}(\ln S)^{2}}\right]
$$

Where $J_{0}$ is nucleation constant, $\gamma$ is surface energy density, $v$ is partial molar volume of the transition metal hydroxide precipitate, $k_{\mathrm{B}}$ is boltzmann's constant, $R_{\mathrm{g}}$ is universal gas constant, $T$ is temperature in Kelvin scale, $S$ is supersaturation ratio of the precipitations. Thus, in the precursor growth process, the ammonia concentration increases gradually, according to equations (1), (2) and (3), it can be judged that the $\mathrm{NH}_{3}-\mathrm{TM}$ complex gradually increases and $\mathrm{TM}(\mathrm{OH})_{2}$ precipitation amount decreases, resulting in the decrease of precipitation supersaturation, the decrease of nucleation rate and the increase of growth rate (principle of mass 
conservation). Additionally, a focused beam reflective measurement (FBRM) is used to obtain the change information of particle number and size in process analysis, as exhibited in Fig. 1d-e. It can be seen from Fig. 1d that the following phenomena take place in the reactor: (1) after the feed liquid continues to enter the reactor, because hydroxide precipitation reaction rate is very fast, a large number of $\mathrm{Ni}_{0.92} \mathrm{Co}_{0.04} \mathrm{Mn}_{0.04}(\mathrm{OH})_{2}$ precipitates increase instantaneously, resulting in a rapid increase of supersaturation, and finally a large number of crystal nuclei are produced rapidly. In the case of strong stirring, the crystal nuclei with high surface energy tend to aggregate to form large irregular agglomerates to release energy rapidly, so the number of microns and nanoparticles increases sharply (zone A); (2) when a certain reaction time is reached, the $\mathrm{TM}(\mathrm{OH})_{2}$ slurry in the reactor starts to flow out from the overflow port, resulting in a slow increase in the number of particles, in addition, small particles grow gradually (zone B); (3) with the increase of reaction time, partial small particles dissolve-aggregate and large particles continue to grow (zone C); (4) with the continuous addition of solution in the reactor, the large particles begin to dissolve-recrystallize-aggregate under a certain stirring strength, and finally the small particles increase and the large particles decrease (zone D); (5) the system reaches steady state after the growth rate and nucleation rate of precursor are stable, and the particle size is relatively stable (zone E), as displayed in Fig. 1e (Fig. 1e is a partial enlarged drawing of zone E). 

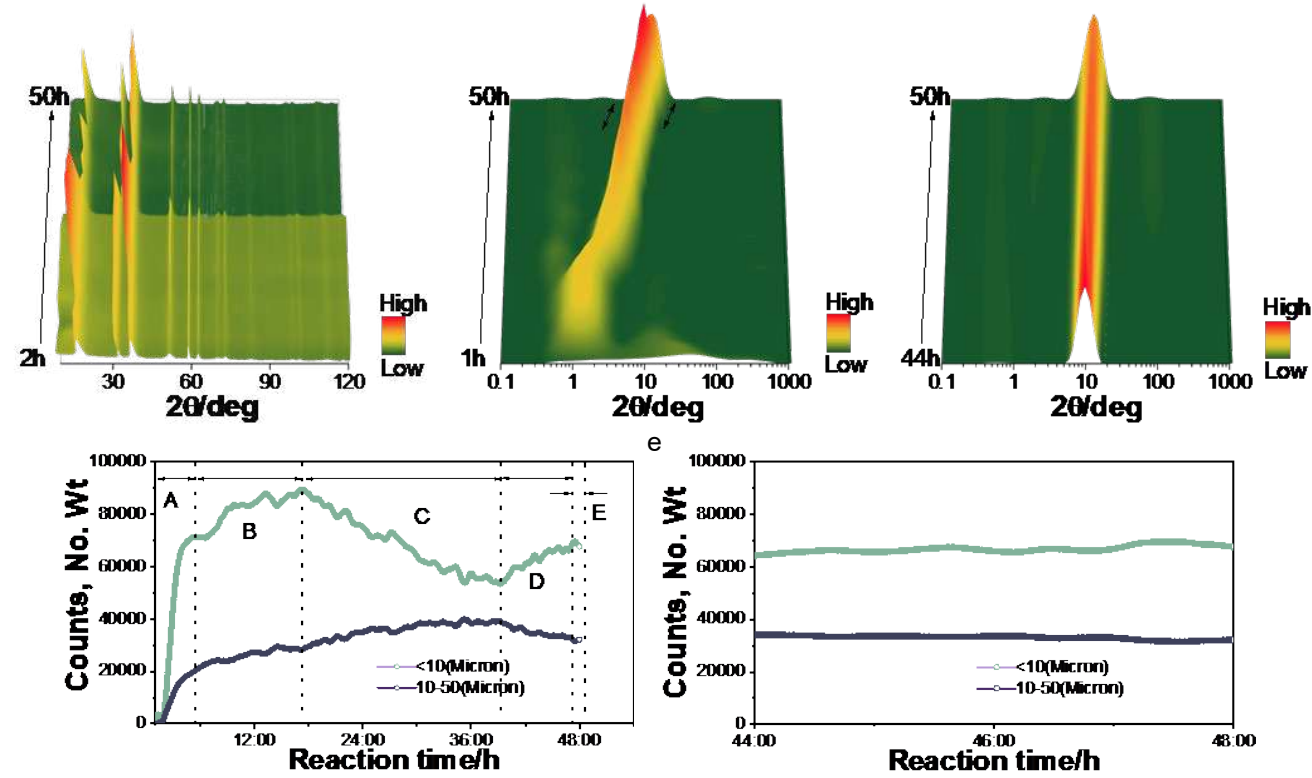

Fig. 1. Observation on the structure, change of particle size and number in the process of precursor synthesis. (a) XRD patterns and (b, c) particle size distribution curve of $\mathrm{Ni}_{0.92} \mathrm{Co} 0.04 \mathrm{Mn}_{0.04}(\mathrm{OH})_{2}$; (d, e) Changes of FBRM total counts during coprecipitation process (A3 sample).

SEM is used to observe precursor morphological characteristic to track and analyze the particle morphology. In the initial stage of coprecipitation reaction, small particles tend to gather rapidly to form aggregations. Therewith, part of $\mathrm{TM}(\mathrm{OH})_{2}$ precipitations is used for nucleation and part for growth, which make the original small particles grow gradually, as shown in Fig. 2a-k. Fig. 2b-h show the morphology of precursor primary particles is hexagonal sheet structure. It is mentioned in the previous literature that precursor crystal grows mainly along the [001] direction. ${ }^{[27]}$ Generally, the [001] facet contains (100), (010) and (110) crystal faces, the inter-plane spacing of each crystal plane is equal, and the angle between any of crystal planes is $60^{\circ}$. Therefore, the primary particle morphology is hexagonal nanoplate (the crystal structure is shown in Fig. S4). Especially, the primary particles of precursors under different reaction conditions also show hexagonal nanosheets, as displayed in Fig. S5-7. As the reaction proceeds, the primary particles have anisotropic growth, and the hexagonal morphology gradually disappears. Notably, Fig. 2k-p display primary particle size decreases gradually from $32 \mathrm{~h}$ to $44 \mathrm{~h}$, and continues to grow from $44 \mathrm{~h}$ to $50 \mathrm{~h}$. The main reason is that due to the secondary nucleation on the surface of the 
particles, the newly generated nuclei continue to grow and cover on the original primary particles, so that the average particle sizes of the overall primary particles decrease. The original primary particles are completely covered until the growth reaches a certain stage, and finally the newly generated primary particle size is displayed. As shown in Fig. S5 (A1 sample), it can be clearly seen that new small particles grow on the surface of primary particles with $32 \mathrm{~h}$ reaction time. Additionally, the samples under different reaction conditions also show the same primary particles size change trend, which also proves the existence of secondary nucleation in the precursor growth process, as displayed in Fig. S6, 7. Fig. 2r shows the change trend of A3 sample primary particles size under the different reaction time. Especially, Fig. 2 and Fig. S5-7 demonstrate the thickness of the hexagonal nanoplate increases first and then decreases. As mentioned earlier, the crystal planes on the side of the nanosheets contain (100) faces. It is suggested that the peak intensity of (100) crystal planes should increase first and then decrease, which is consistent with the change of (100) peak strength in Fig. 1a. 

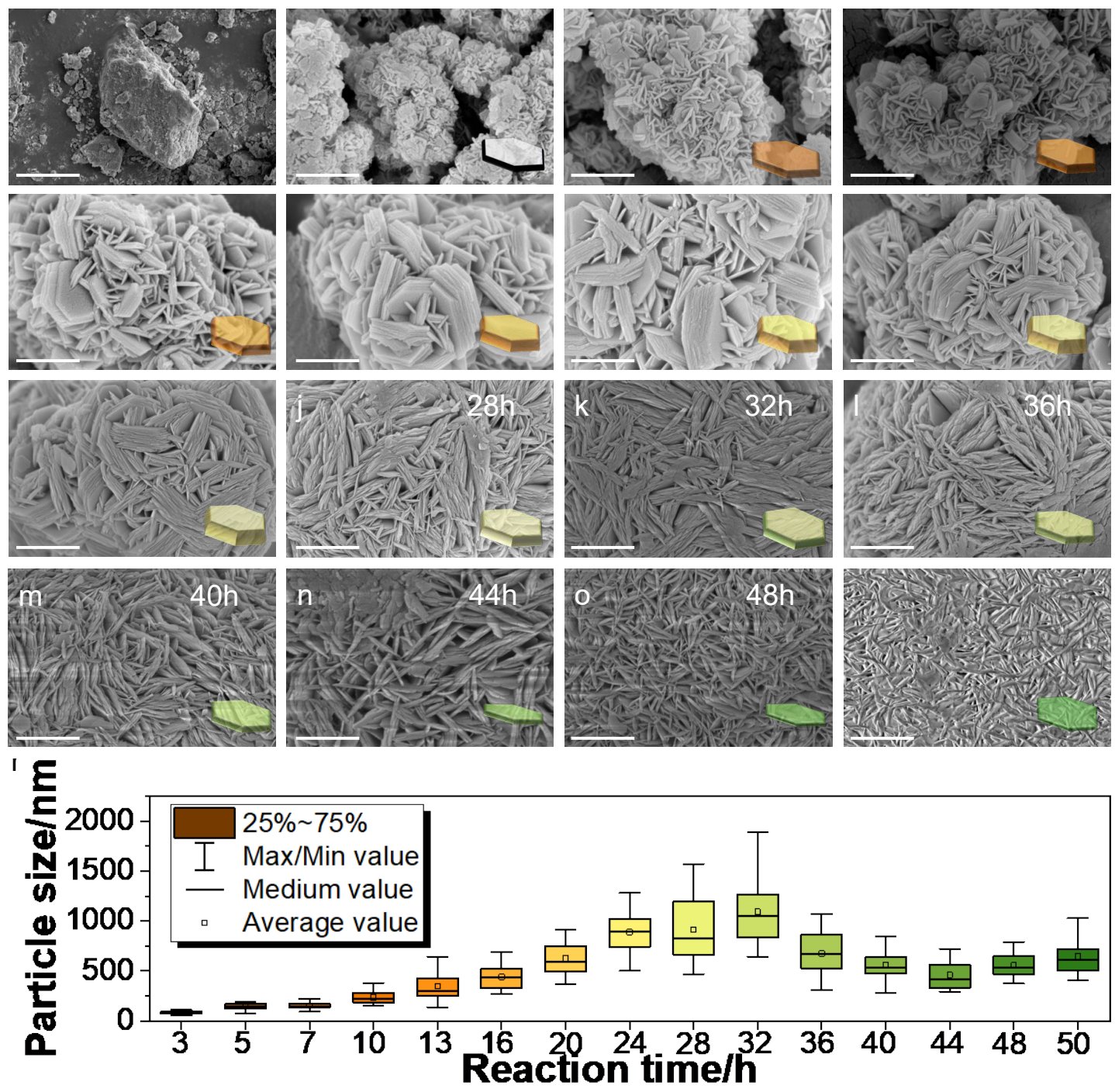

Fig. 2. Observations of precursor morphology. (a-p) SEM images and (r) primary particle size statistics of $\mathrm{Ni}_{0.92} \mathrm{Co}_{0.04} \mathrm{Mn}_{0.04}(\mathrm{OH})_{2}$ in various time (A3 sample). Scale bars, $10 \mu \mathrm{m}$ (a), $500 \mathrm{~nm}(\mathrm{~b}-\mathrm{g}), 1 \mu \mathrm{m}$ (h-p).

In the process of precursor synthesis, the element precipitation uniformity is also a key factor for cathodes electrochemical performances. ${ }^{[28]}$ Therefore, cross-sectional SEM and HAADF-EDS are used to detect the homogeneity of precursor element precipitation. Fig. S8 shows that the primary particle displays elongated nanosheet. The elements distribution on the surface and inside of the precursor is uniform in the microscale, as exhibited in Fig. S9, 10. The distribution of precursor elements is still uniform in the nanoscale, but the elements proportions in different parts of precursor are different, as exhibited in Fig. S11. The Mn concentration in the middle and core regions of precursor is higher than that in the outer region. 
Furthermore, TEM is used to detect the microstructure of the precursor. Fig. 3a displays that the surface structure of the precursor belongs to $\mathrm{Ni}(\mathrm{OH})_{2}$ with the space group $\mathrm{P} \overline{3} \mathrm{~m} 1$ ((100) and (101) crystal faces) and $\mathrm{MnOOH}$ with the space group $\mathrm{P} 21 / \mathrm{c}$ ((3-2-3) crystal face). Fig. 3b, c show the microstructure in the middle and core of the precursor. The B1, B2, C1 and $\mathrm{C} 2$ regions of Fig. 3b, c show a twinned crystal, which are mainly composed of $(001)$ crystal face of $\mathrm{Ni}(\mathrm{OH})_{2}(\mathrm{P} \overline{3} \mathrm{ml})$ and $(100)$ crystal face of $\mathrm{MnOOH}\left(\mathrm{P}_{1} / \mathrm{c}\right)$. From a thermodynamic point of view, compared the Gibbs free energy of $\mathrm{MnO}(-1.97 \mathrm{eV})$ and $\mathrm{Mn}_{2} \mathrm{O}_{3}(-2.02 \mathrm{eV}), \mathrm{Mn}^{2+}$ is more easily oxidized to $\mathrm{Mn}^{3+}$ in precursor growth process in contact with relative oxygen-rich environment. ${ }^{[9]}$ Therefore, $\mathrm{MnOOH}$ phase is easy to form in the coprecipitation process. Additionally, as shown in the A2 area of Fig. 3a, the crystal structure of $\mathrm{Ni}(\mathrm{OH})_{2}$ phase is different from that of $\mathrm{MnOOH}$ phase, which leads to local lattice mismatch. Especially, Fig. 3b, c show that there is an epitaxial relationship between $\mathrm{Ni}(\mathrm{OH})_{2}$ phase and $\mathrm{MnOOH}$ phase, where (001) plane of $\mathrm{Ni}(\mathrm{OH})_{2}$ phase and (100) plane of $\mathrm{MnOOH}$ phase are parallel to each other. Generally, $\mathrm{Ni}(\mathrm{OH})_{2}$ phase and $\mathrm{MnOOH}$ phase are not compatible, and their molar volumes are different (the molar volumes of $\mathrm{MnOOH}$ : $24.06 \mathrm{~cm}^{3} \mathrm{~mol}^{-1}$, the molar volumes of $\left.\mathrm{Ni}(\mathrm{OH})_{2}: 23.47 \mathrm{~cm}^{3} \mathrm{~mol}^{-1}\right) \cdot{ }^{[9,29]}$ To adapt the internal strain caused by the nucleus produced via $\mathrm{MnOOH}, \mathrm{MnOOH}$ phase constructs a twinned structure along [001] direction, thus $\mathrm{Ni}(\mathrm{OH})_{2}$ maintains an epitaxial structure with $\mathrm{MnOOH}$, as shown in Fig. S12. 

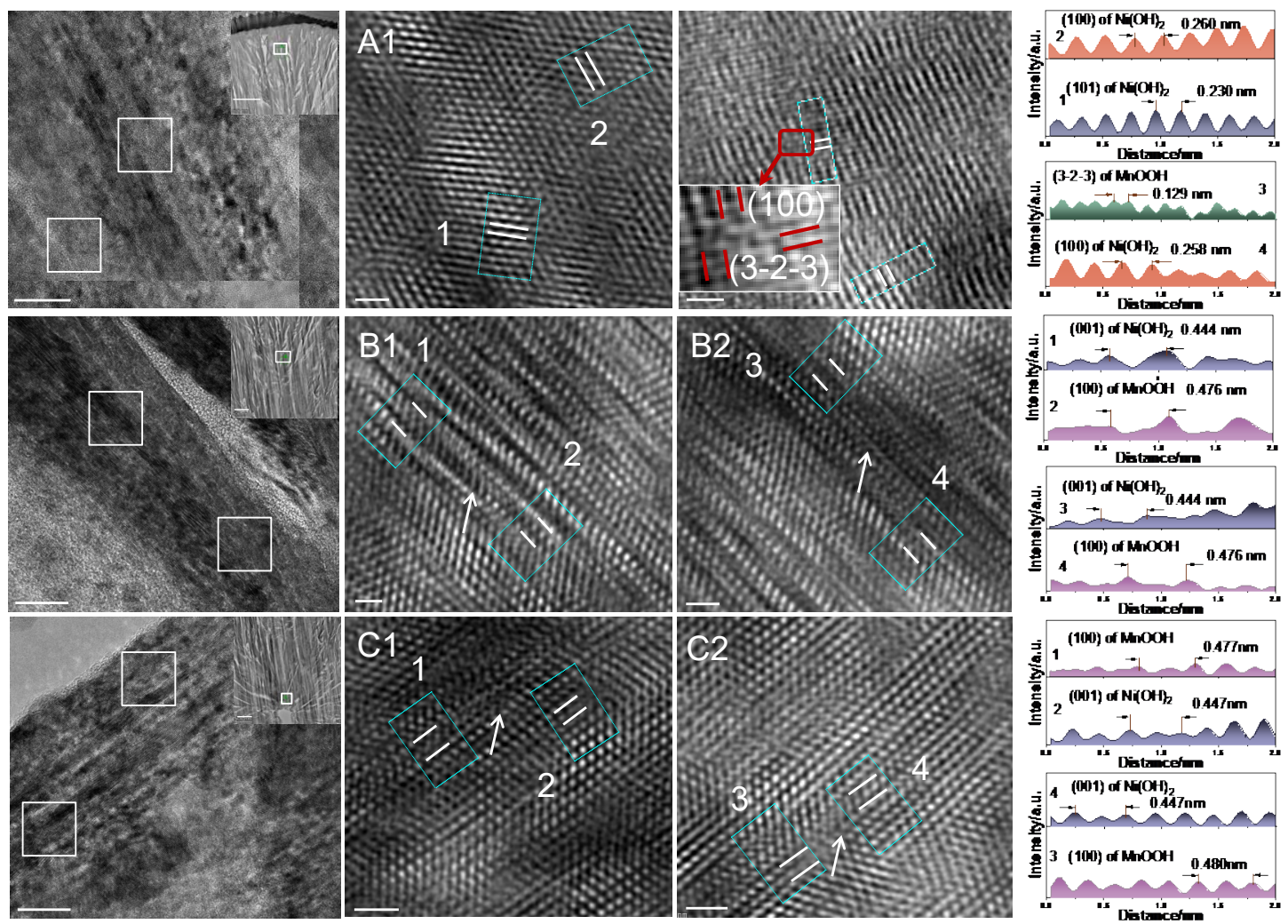

Fig. 3. Observations of precursor microstructures. (a-c) The cross-sectional HRTEM images of $\mathrm{Ni} 0.92 \mathrm{Co} 0.04 \mathrm{Mn} 0.04(\mathrm{OH})_{2}$ precursor, the insert image presents low magnification cross-sectional HRTEM (A3 sample). Scale bars, $10 \mathrm{~nm}$ (a-c), $500 \mathrm{~nm}$ (the inset in (a-c)), $1 \mathrm{~nm}((\mathrm{a}-\mathrm{c})$ corresponding local amplification region).

\section{Primary particle size regulation}

The control of precursor structure is determined by process conditions and reactor. Therefore, it is very important to understand the impact of relevant parameters. For hydroxide coprecipitation process: (1) ammonia concentration and $\mathrm{pH}$ value will affect the complexation (equation (1)) and precipitation reaction (equation (2)), which causes the equilibrium of precipitation reaction to move to the left or right; (2) stirring speed will affect the mass transfer coefficient $(k)$, resulting in the overall shift of $(1,2)$ reaction equilibrium. For mass transfer process, Sherwood number (Sh), Reynolds number $(R e)$ and Schmidt number $(S c)$ generally have the following correlations: ${ }^{[30]}$

$$
\begin{gathered}
S h=2+A R e^{m} S c^{n} \\
S h=\frac{k \mathrm{~d}}{\mathrm{D}}
\end{gathered}
$$

$A, m$ and $n$ are constants, $\mathrm{d}$ is the average particle size of sotel, $\mathrm{D}$ is reactant diffusion 
coefficient. Therefore, with the increase of stirring speed, the larger the Reynolds number is, the larger Sherwood number is, the larger the mass transfer coefficient is, resulting in the increase of supersaturation and nucleation rate, and the decrease of growth rate. Finally, primary particles size decrease with the increase of stirring speed, vice versa. Based on the understanding of the influence of ammonia concentration and stirring speed on the reaction process, we realize the effective control of the primary particle size of the precursor. Fig. S13 shows the primary particle size and precursor morphology under different reaction conditions (A1 sample: $182 \mathrm{~nm}$; A2 sample: 433 nm; A3 sample: $520 \mathrm{~nm}$, A4 sample: $255 \mathrm{~nm}$ ). Notably, the research on the precursor growth mechanism demonstrates the secondary nucleation existed in the process of coprecipitation, which led to the increase of primary particle size first and then decrease, finally increase. Especially, the peak value of primary particle size appears $\sim 32 \mathrm{~h}$ (before secondary nucleation). Based on the above exploration, the enhanced primary particle size can be achieved by inhibiting secondary nucleation, yet the secondary particle size may become smaller.

In addition, the fluid flow state in the reactor also plays a key role in the precursor structure regulation. Therefore, the designed impeller structure is adopted to reduce the collision rate between particles and impeller to inhibit the secondary nucleation. ${ }^{[31]}$ Fig. 4a exhibits the fluid flow simulation of the conventional and designed impeller, which shows that the impeller with special structure can effectively reduce the shear rate. The reduction of shear rate can effectively decrease the collision rate between particles and impeller. The designed impeller is used for coprecipitation reaction. After reaction for $50 \mathrm{~h}$ (A5 sample), the secondary (primary) particle size is $\sim 6 \mu \mathrm{m}(\sim 670 \mathrm{~nm})$ as displayed in Fig. 4b-f. Importantly, after the reaction time reached $24 \mathrm{~h}$, the primary particle sizes do not change obviously, indicating that the secondary nucleation is effectively inhibited (Fig. 4g). 

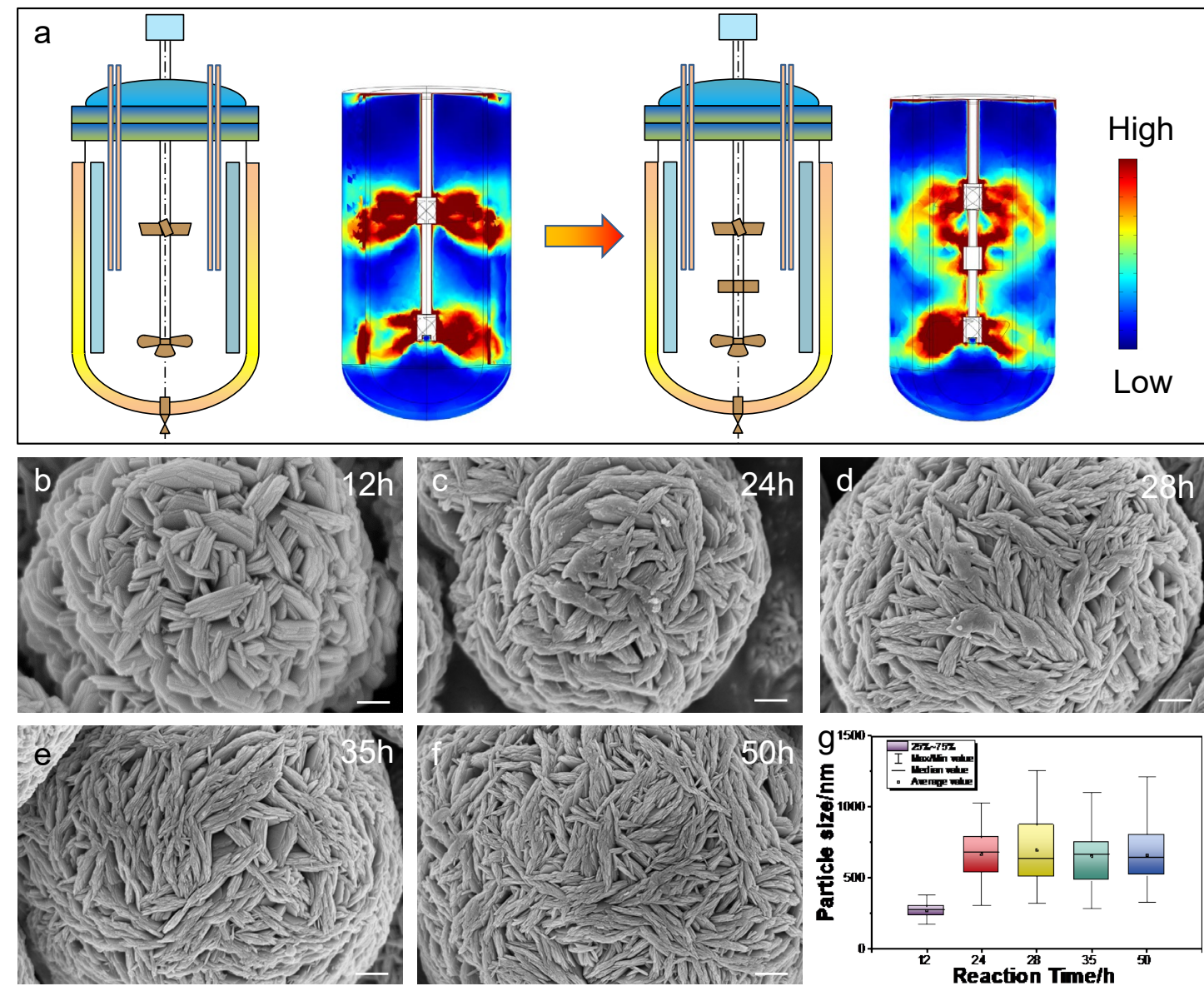

Fig. 4. The reactor flow field distribution and observations of precursor morphology. (a) Shear rate distribution. (b-f) SEM images of $\mathrm{Ni}_{0.92} \mathrm{Co}_{0.04} \mathrm{Mn}_{0.04}(\mathrm{OH})_{2}$ (A3 sample) in different reaction time. (g) Primary particle size statistics. Scale bars, $200 \mathrm{~nm}(\mathrm{~b}), 500 \mathrm{~nm}(\mathrm{c}-\mathrm{f})$.

\section{Discrepancy of primary particle sizes on cathodes structure degradation}

To analyze the influence of primary particles size on structure degradation, the electrochemical performance of A1, A2 and A3 samples (secondary particle size: 14 $\mu \mathrm{m})$ are tested in the voltage window of 2.7-4.3 V. The main reason for selecting samples A1, A2 and A3 is to eliminate the influence of secondary particle size on electrochemical performance as much as possible, the distribution of the precursor size is shown in Fig. S14. Fig. 5a-c display the cathodes morphology for the A1, A2 and A3 precursors after high-temperature lithiation, which shows that the primary particles of the precursor change from flake to polyhedron via high temperature calcination, and its size is similar to the original. Fig. $5 \mathrm{~d}$ shows that the primary particle size of A3 sample after sintering is the largest (A1 (336 nm) vs A2 (447 nm) 
vs A3 (565 nm)). Additionally, the calcined materials show that the cell parameter $c / a$ value is greater than 4.9, and the TEM characterization shows a clear (003) crystal plane for bulk and surface structure, which indicates that these samples lithiated at $745^{\circ} \mathrm{C}$ display a good R-3m layered structure, as exhibited in Fig. S15 and Table S3-5. Fig. 5e demonstrates that the structural stability of A3 sample is obviously better than that of A1 and A2 samples after 200 cycles at $0.5 \mathrm{C}$ (69.5\% (A3) vs $61.3 \%$ (A2), 51.2\% (A1)), and the corresponding charge-discharge curves are shown in Fig. S16. Especially, for the dense accumulation of secondary particles, intergranular crack is one of the important factors of structure attenuation. It is clearly shown in Fig. 5f-h that the cracking degree of A3 sample is lighter than those of A1 and A2 samples. Additionally, it can be seen from electrode flake section that A1 and A2 samples are more seriously powdered than A3 sample. At the same time, Fig. S17 displays the secondary particles with smaller primary particles are seriously powdered after cycling. Besides, intragranular crack is also an important factor for the structural degradation of the cathodes. It can be clearly recognized that intragranular cracks of A3 sample are less than those of A1 and A2 samples as the cross-section SEM shown in Fig. 51-n, the yellow arrow indicate viods and the red arrow indicate cracks. Notabbly, the top illustration in Fig. 5f-h also shows that the primary particle cracking degree of A3 sample is lighter than those of A1 and A2 samples. These results indicate that larger primary particles can inhibit the generation of intergranular and intragranular cracks. Additionally, it is also generally accepted that the performance decay is due to the side reaction at the interface between electrolyte and cathodes, which will form a thick electrochemical inertness layer (CEI film) on the surface and seriously corrode the cathodes surface. The main reaction process is as follows (M: transition metal ion): $:^{[18]}$

$$
\begin{gathered}
\mathrm{HF}+\mathrm{PF}_{6}{ }^{-}+\mathrm{ROCO}_{2} \mathrm{R}+\mathrm{M}+\mathrm{Li}_{2} \mathrm{O} \rightarrow \underset{\mathrm{LiF}}{ }+\mathrm{Li}_{x} \mathrm{PF}_{y}+\mathrm{Li}_{x} \mathrm{PF}_{y} \mathrm{O}_{z}+\mathrm{M}_{n} \mathrm{~F}+\mathrm{ROCO}_{2} \mathrm{Li}+ \\
\mathrm{Li}_{x} \mathrm{CO}_{3}+\mathrm{LiOH}
\end{gathered}
$$

Fig. 5o-q demonstrate that the thickness of CEI film with larger primary particles (A3: $3.5-4.1 \mathrm{~nm}$ ) is obviously thinner than that with smaller primary particles (A1, A2: 23 - $30 \mathrm{~nm}, 5.5-6.4 \mathrm{~nm}$, respectively), which is mainly due to the larger specific surface 
area of small particles (the larger the specific surface area is, the larger the active surface is).

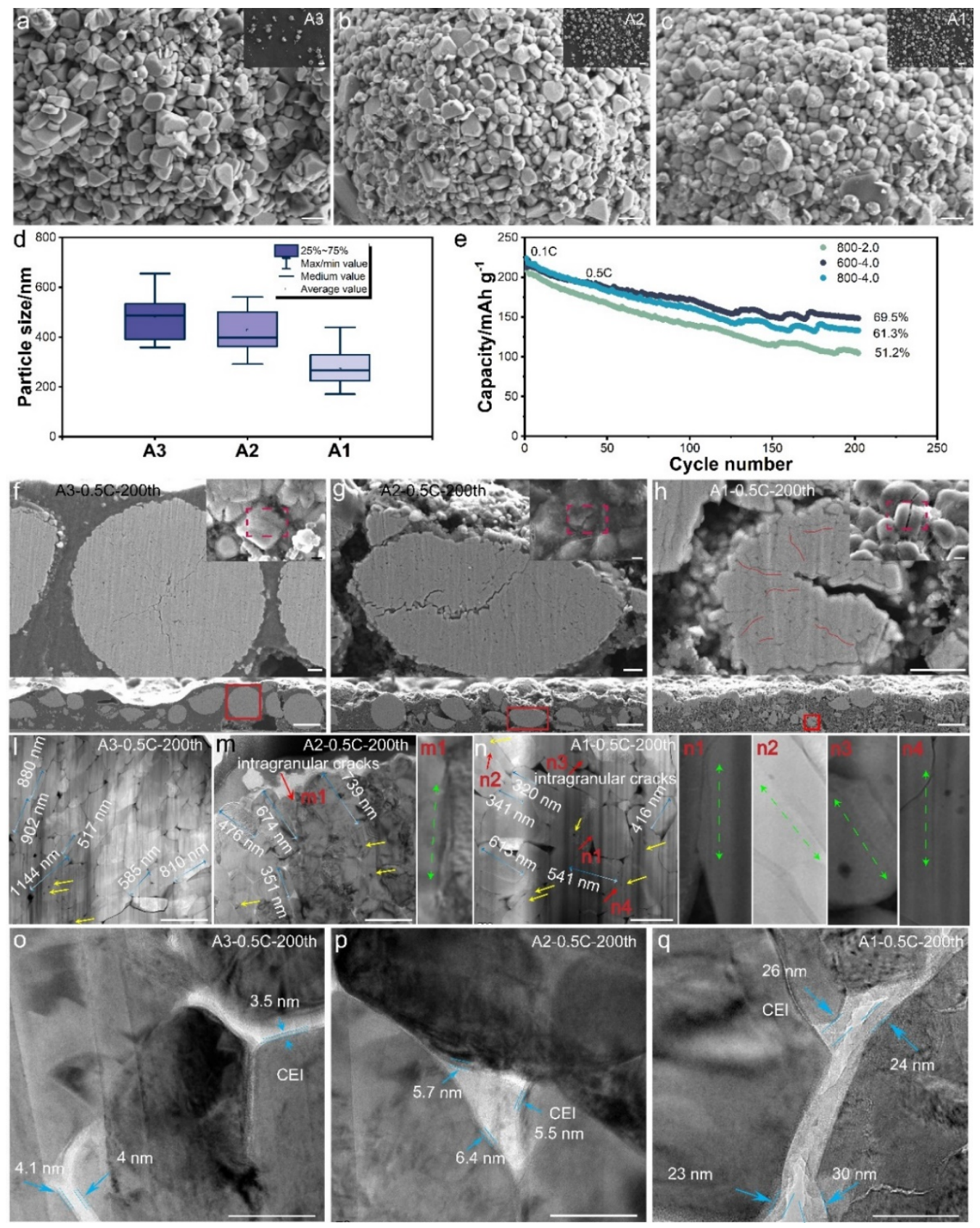

Fig. 5. Electrochemical performance, observations of morphology, CEI films \&

cracks. $\mathrm{LiNi}_{0.92} \mathrm{Co}_{0.04} \mathrm{Mn}_{0.04} \mathrm{O}_{2}$ SEM images (a-c), particle sizes statistics (d), cycling performance (e), cross-section HAADF / SEM images after 200 cycles at $0.5 \mathrm{C}$ (f-q). The top illustration shows the particle surface, and the bottom is the electrode flake section in (f-h). The g1 and h1-4 are a partial enlarged view of the corresponding marked position. Scale bars, $500 \mathrm{~nm}$ (a-c, m, n), $1 \mu \mathrm{m}$ (f-1), $100 \mathrm{~nm}$ (the top inset (f-h) and (o-q)), $20 \mu \mathrm{m}$ (inset (a-c)), $10 \mu \mathrm{m}$ (the bottom inset (f-h)). 
During the cycling process, the loss of $\mathrm{Li}$ and $\mathrm{O}$ in cathodes will lead to the transformation from layered structure to spinel / rock salt phase, which will hinder the diffusion of lithium ions. The structure degradation equation of charge-discharge process can be expressed as follows: ${ }^{[32]}$

$$
\begin{gathered}
\mathrm{Li}_{x} \mathrm{NiO}_{2} \rightarrow(1+x) \mathrm{Li}_{x /(1+x)} \mathrm{Ni}_{1 /(1+x)} \mathrm{O}+(1-x) / 2 \mathrm{O}_{2} \\
\mathrm{Li}_{x} \mathrm{NiO}_{2} \rightarrow\left[x / 2-\delta /(2-2 \delta) \mathrm{Li}_{2} \mathrm{O}+1 /(1-\delta) \mathrm{Li}_{\delta} \mathrm{Ni}_{1-\delta} \mathrm{O}+(2-3 \delta-x+\delta x) /(4-4 \delta) \mathrm{O}_{2}\right.
\end{gathered}
$$

In addition, $\mathrm{Ni}^{2+}$ ions $(0.69 \AA)$ are easy to migrate from the transition metal layers to the lithium layers because of its similar radius to $\mathrm{Li}^{+}$ions $(0.76 \AA)$, resulting in the structure degradation of particle surface (layered structure - spinel / rock salt structure). ${ }^{[33]}$ Fig. $6 \mathrm{~d}$ shows a schematic diagram of the structural transformation caused by lithium ion migration. To further analyze the structure decay of different primary particles size during cycling, TEM is used to detect the microstructure. Fig. 6a exhibits the interior (site B) and exterior (site A) of primary particle are composed of rock salt and spinel phases, indicating the loss of lithium and oxygen in small primary particles and the migration of $\mathrm{Ni}$ ions to the lithium layer are very serious during the charging / discharging process. Fig. $6 \mathrm{~b}$ displays the surface structures are spinel and rock salt phase, but only spinel phase is contained in the interior. Compared with spinel phase, rock salt phase has greater resistance to lithium ion migration. Therefore, the electrichemical performance of A2 sample is better than that of A1 sample. Particularly, the surface structure of cathode (A3) is rock salt phase, but the internal layered structure maintains integrity as shown in Fig. 6c, which indicates the primary particle with the larger size has a better structure stability. 


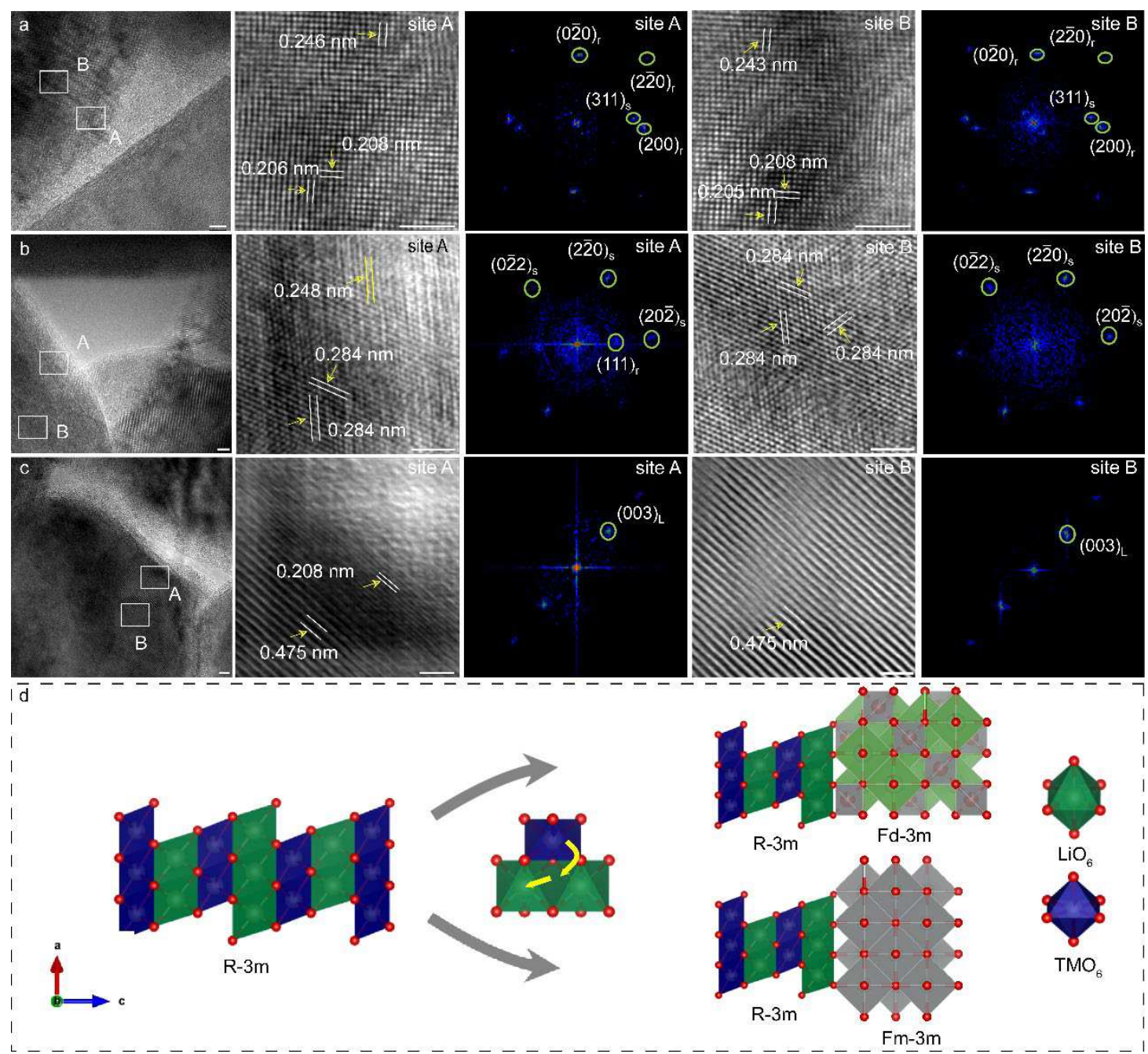

Fig. 6. Observations of microstructures after 200 cycles at $0.5 \mathrm{C}$. Cross section high resolution TEM images and corresponding FFT of (a) A1, (b) A2 and (c) A3 samples. The structure decay model (d). Scale bars, $10 \mathrm{~nm}$ (a-c), $2 \mathrm{~nm}$ (local enlarged drawings of corresponding area in (a-c)).

Intragranular crack is the key factor to the performance degradation. ${ }^{[13]}$ Usually, the electrolyte will penetrate along the crack and corrode the active material, resulting in the deterioration of electrochemical performance. In order to further elaborate the degradation of layered structure (R-3m) and the internal structure of intragranular cracks, we analyze the microstructure of primary particles inside the secondary particles after cycling. Two types of cracks can be identified from the Fig. 7a, b. Fig. 7a demonstrates that type I crack possesses narrow, dark stripes (area indicated by blue arrow). The dark stripe is parallel to (003) plane, and its plane spacing $(0.79 \mathrm{~nm})$ is larger than (003) plane $(0.48 \mathrm{~nm})$. Especially, the interior of dark stripe is not empty 
(area indicated by green arrow), but has some structural features with rock salt phase, and the tip of dark stripe presents a "V-shape". The internal structure of dark stripe and its low density atoms indicate the loss of lithium and oxygen, and also demonstrate dark stripe is formed by the splitting of (003) crystal plane. The crystal model is shown in Fig. 7d. The other type is the classical crack with two free surfaces (Fig. 7b). Besides the two crack tips (there is a "V-shape" at the tip), the two free surfaces are parallel, which is obviously different from intergranular cracks caused by rapid stress changes. Moreover, crack is significantly parallel to (003) plane of cathode (layered structure). Notably, the tip of dark stripe also presents a "V-shape" in Fig. $7 \mathrm{a}$, which indicates that it is the early stage of the classical crack with two free surfaces (premature crack). Furthermore, crack at site A in Fig. $7 \mathrm{~b}$ confirms this conjecture. The edge dislocation can also be observed from Fig. 7a, b the area indicated by the red arrow). At the core area of dislocation, the lattice distortion and mismatch will lead to strain enhancement. ${ }^{[13]}$ Especially, dislocation has been reported as a fast channel for ionic transport. ${ }^{[34]}$ Attentively, Fig. 7a, b can be clearly observed that the edge dislocations are associated with the premature cracks, which implies that the dislocation core is the nucleation of premature cracks. Combining the characteristics of dislocations with the viewpoint of energy, Li and $\mathrm{O}$ at dislocations area are lost preferentially in order to release strain. Additionally, from the kinetical point of view, the dislocation region is also a fast diffusion channel. Therefore, the edge dislocation core can be used as the nucleation site of crack formation. In the process of lithium ion intercalation and extraction, the strain can not be released in time, which leads to dislocation formation, and the structure deteriorates in the subsequent cycle. Fig. 7c further proves that the layered structure of A1 sample is seriously damaged, where both the interior and exterior structures are rock salt phases. Fig. S18 shows the different primary particle structure changes of A2 sample. Fig. S18a shows that the surface and internal structures of the particle are rock salt phase and layered structure respectively, which indicates that the particle structure is relatively stable compared with that in A1 sample. Fig. S18b, c display that the premature crack is also observed in the primary particle, and their structures are 
connected with dislocations in space, which also demonstrates that dislocations can be used as nucleation sites for the crack initiation. In order to further verify the influence of cathode primary particle size on the structure degradation, the microstructure of different primary particles in A3 sample is also analyzed. Fig. S19 shows that primary particles structures are more stable than those of A1 and A2 samples. Here, the surface and internal structure of particles are rock salt phase and layered structure, respectively. In particular, edge dislocations can be clearly observed in the Fig. S19, but premature cracks are rarely observed. It can be clearly seen that the generation of intragranular cracks is effectively restrained when the primary particle size is larger. As observed in Fig. 5f-n, A3 sample possesses fewer intragranular cracks. Therefore, the larger primary particles have better structural stability, which may be due to the smaller dislocation nucleation kinetics of larger particles. 


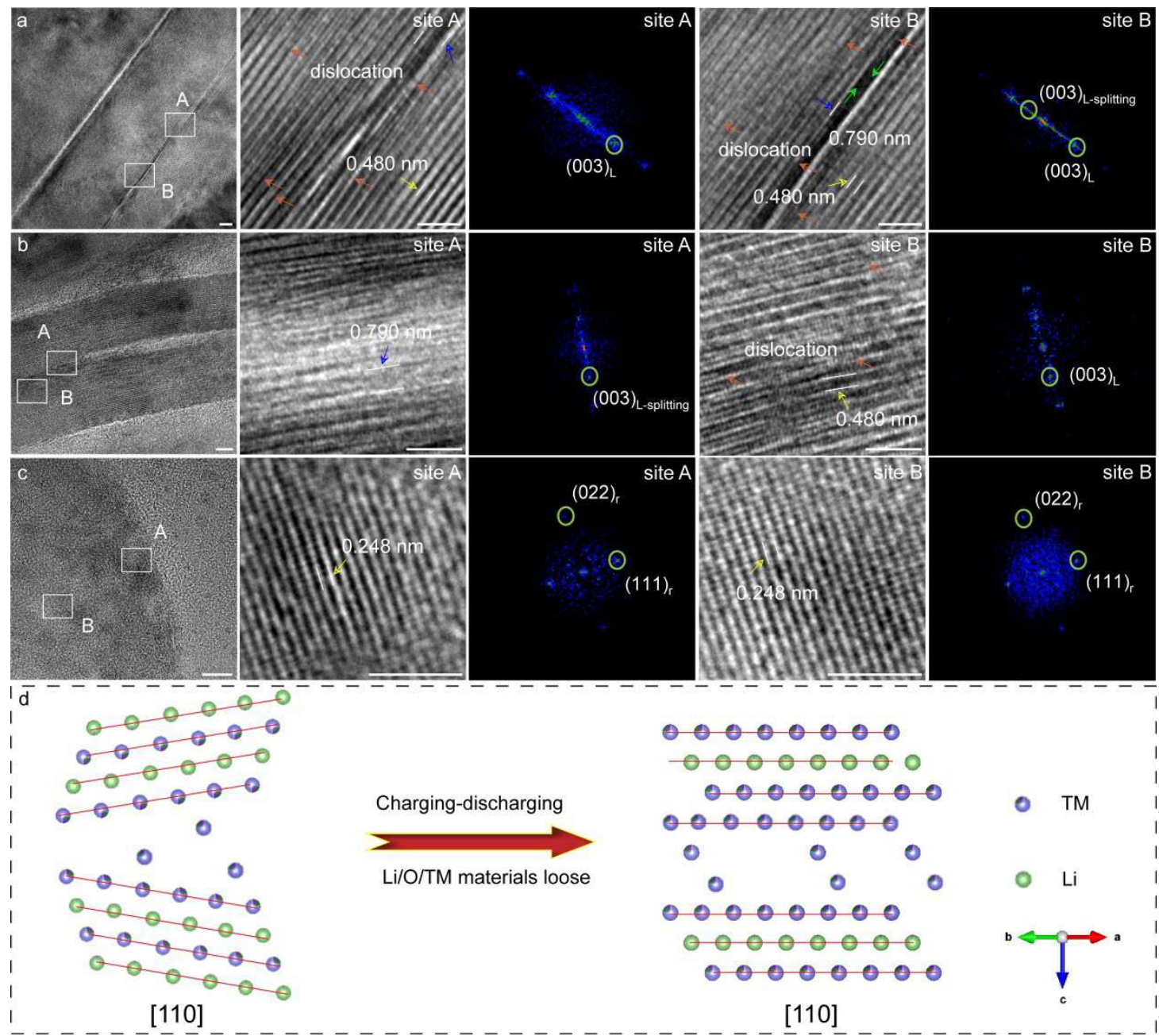

Fig. 7. Observations of intragranular cracks, dislocations and microstructures

after 200 cycles at 0.5 C. Cross section high resolution TEM images and corresponding FFT of A1 sample (a-c), cracking model (d). Scale bars, $10 \mathrm{~nm}$ (a-c), 2 $\mathrm{nm}$ (local enlarged drawings of corresponding area in (a-c)).

\section{Structure stability research and cathodes development direction prediction}

To deeply elaborate the effect of primary particle size on the structure degradation, the stress distribution of $\mathrm{A} 1$ and $\mathrm{A} 3$ particles are calculated. Fig. 8a, b show the stress distribution in the secondary particles of $\mathrm{A} 1$ and $\mathrm{A} 3$ samples during the fully charged state (delithiation). The stress between primary particles will lead to the nucleation of cracks. It is shown here that the stress of A1 sample is larger than that of A3 sample at the boundary, indicating that larger size primary particles can inhibit the intergranular cracks formation. Additionally, Fig. 8c-f show the stress distribution of primary particles along (003) crystal plane direction. It is shown that the stress along the (003) crystal plane of smaller primary particles is difficult to return to the original state 
compared with that of larger primary particles, indicating the smaller primary particles are easier to crack along the (003) crystal plane. These results show that the polycrystalline agglomerated particles with larger primary particles size can effectively inhibit the intergranular and intragranular cracks formation, thus improving the structural stability of the material. Additionally, Fig. S20 demonstrates the H2-H3 peak intensity of A3 sample still has a certain intensity after 200 cycles, which is compared with other samples (A1, A2). The H2-H3 phase transition process causes the drastic shrinkage change along the $c$-axis direction, which is mainly related to the sharp shrinkage of the interlayer distances between $\mathrm{NiO}_{2}$ due to the oxidation of $\mathrm{Ni}^{2+}$ to $\mathrm{Ni}^{4+}$ in the octahedral sites $(3 \mathrm{a}, 3 \mathrm{~b})$. Especially, compared with $\mathrm{A} 1$ and $\mathrm{A} 2$ samples, there is only a small change in the peak intensity of H1-M for A3. These features indicate that the structure degradation of A1 and A2 samples is more serious than that of A3 sample. In situ high-temperature XRD (HT-XRD) means is carried out to estimate the structural stability of A1 and A3 samples at charging $4.3 \mathrm{~V}$. Fig. $8 \mathrm{~g}$ displays that the initial layered oxide structures with the space group of R-3m of A1 and A3 samples are preserved after charging to $4.3 \mathrm{~V}$. As the temperature rises to $\sim 200{ }^{\circ} \mathrm{C}$, the A1 sample' structure converted into a disordered spinel structure with the space group of Fd-3m as (108)L and (110)L peaks merge into (440)s peak (Fd-3m). Subsequently, the overall structure is transformed into rock salt phase (Fm-3m) as the characteristic peaks (111)r and (220)r appear when the temperature reaches $\sim 480{ }^{\circ} \mathrm{C}$. For A3 sample, the phase transition temperatures increase to $\sim 240{ }^{\circ} \mathrm{C}(\mathrm{Fd}-3 \mathrm{~m})$ and $\sim 560{ }^{\circ} \mathrm{C}(\mathrm{Fm}-3 \mathrm{~m})$, respectively. The deferred phase transition demonstrates that $\mathrm{A} 3$ sample has better structural stability than that of A1, i.e. larger cathode' primary particle size can effectively enhance the structural stability. In general, the secondary particle size also affects the cathode cycling performance, what are the dual effects of primary particles and secondary particles? The A5 sample lithiated at $745{ }^{\circ} \mathrm{C}$ is electrochemically characterized. Fig. S21a, b show that the morphology of the precursor after lithiated is spherical, the secondary particle size is $\sim 6 \mu \mathrm{m}$, and the primary particle size is $\sim 675 \mathrm{~nm}$. Fig. S21c exhibits that the cycle retention rate of A5 sample at $1 \mathrm{C}$ is higher than that of $\mathrm{A} 1, \mathrm{~A} 2$ and $\mathrm{A} 3$ samples (63.7\% (A1) vs $66.2 \%$ 
(A2) vs 70.5\% (A3) vs 72.2\% (A5)). These results imply the larger the primary particle is, the smaller the secondary particle is, which can effectively enhance the structural stability. According to the above analysis of the structure stability for primary and secondary particles, we speculate that one of the development directions of Ni-rich cathodes to enhance structure stability is to synthesize quasi single crystal cathodes (secondary particles with agglomeration of multiple larger primary particles), the trend prediction diagram as shown in Fig. 8h.
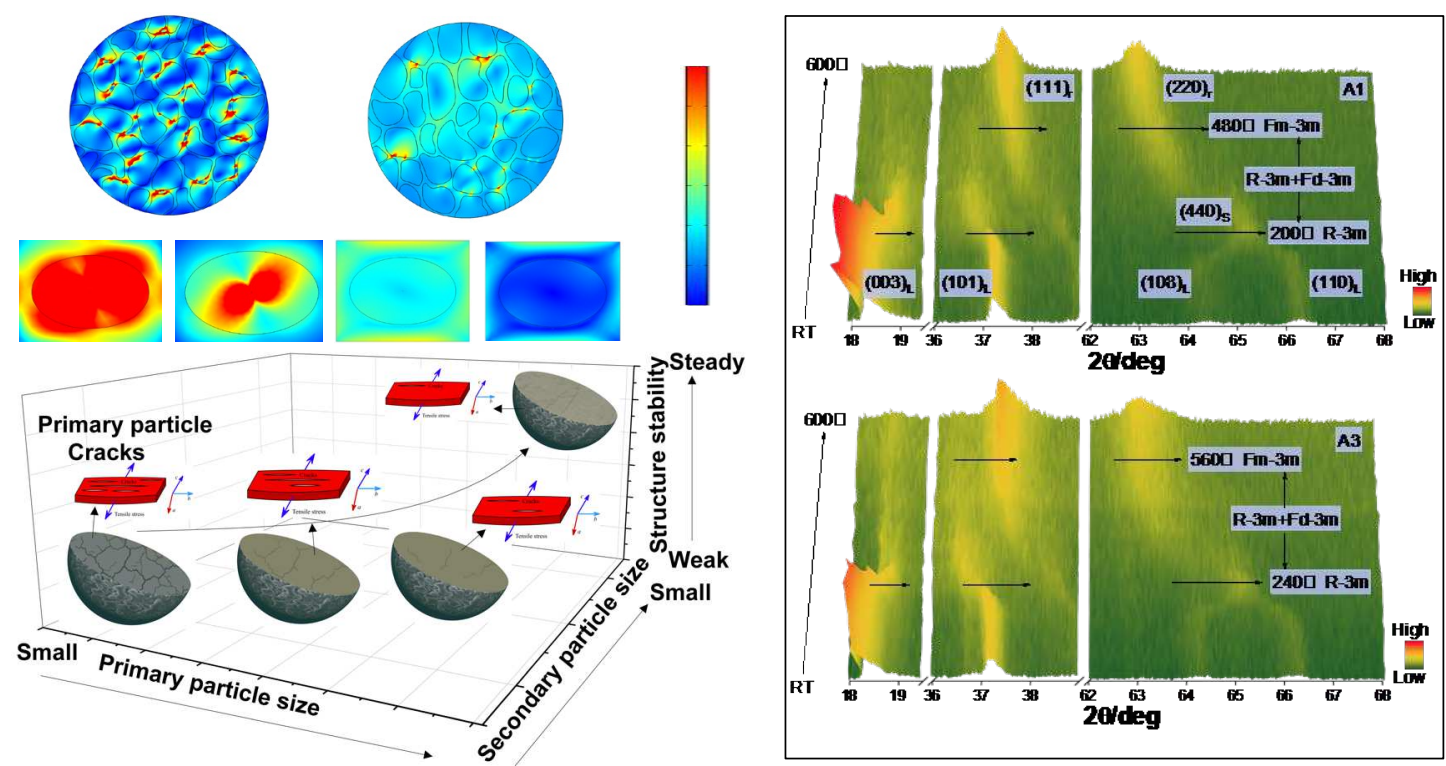

Fig. 8. Particle stress distribution simulation and cathodes development direction

prediction. The stress distribution for (a) A1 and (b) A3 samples at the fully charging. The stress distribution along (003) crystal faces for (c) A1 and (e) A3 at the fully charging, (d) A1 and (f) A3 at the fully discharging. (g) Phase transition temperature of A1 and A3 samples from HT-XRD patterns. (h) Relationship between cathodes structure stability and (primary / secondary) particle sizes.

\section{Discussion}

In this work, the growth and size control of precursor primary particle, and the structural attenuation of cathode primary particle with different size have been systematically studied. In the process of precursor growth, the nucleation rate decreases and the growth rate increases. Additionally, secondary nucleation occurs in this process, and the primary particle size first increases, then decreases, and finally increases. In terms of the precursors microstructures, the precursors show a twinning 
phenomenon, and display $\mathrm{MnOOH}\left(\mathrm{P} 2_{1} / \mathrm{c}\right)$ and $\mathrm{Ni}(\mathrm{OH})_{2}(\mathrm{P} \overline{3} \mathrm{~m} 1)$ phases. Particularly, the increase of ammonia concentration or the decrease of stirring speed will reduce the supersaturation of $\mathrm{TM}(\mathrm{OH})_{2}$ precipitation, resulting in the decrease of nucleation rate, the increase of growth rate and finally the increase of primary particle size. More importantly, optimizing hydrodynamic to control secondary nucleation is used to regulate precursor structure. Inhibiting secondary nucleation can effectively increase the primary particle size and reduce the secondary particle size. During the charge-discharge process, the larger primary particles size can effectively inhibit the structure degradation, CEI film formation and intragranular / intergranular crack formation. Moreover, the larger the primary particle size is, the smaller the secondary particle size is, the better the structural stability of the cathode is. This work provides a basic theoretical reference for the precursor growth mechanism, primary size regulation, and also pointed out the direction for the design of cathode primary particle size.

\section{Methods}

Materials preparation. As our previously reported, the $\mathrm{Ni}_{0.92} \mathrm{Co}_{0.04} \mathrm{Mn}_{0.04}(\mathrm{OH})_{2}$ precursors are synthesized by hydroxide coprecipitation. ${ }^{2}$ A $1 \mathrm{M}$ aqueous solution of stoichiometric mixed $\mathrm{NiSO}_{4} \cdot 6 \mathrm{H}_{2} \mathrm{O}, \quad \mathrm{MnSO}_{4} \cdot \mathrm{H}_{2} \mathrm{O}$ and $\mathrm{CoSO}_{4} \cdot 7 \mathrm{H}_{2} \mathrm{O}$, and a corresponding ammonia concentration aqueous solution $(2.0$ / 4.0 M) are added into a continuous stirred tank reactor at the same feed rate $\left(4 \mathrm{ml} \mathrm{min}^{-1}\right)(\mathrm{CSTR}(5 \mathrm{~L})$, Fig. $\mathrm{S} 1)$. Meanwhile, a $2 \mathrm{M} \mathrm{NaOH}$ solution is used to adjust $\mathrm{pH}$ (11.2) in the cocipitation process. The temperature $\left(50 \pm 1{ }^{\circ} \mathrm{C}\right)$, and stirring speed $(600 / 800 / 1000 \mathrm{rpm})$ are accurately controlled, and the whole reaction process is carried out in $\mathrm{N}_{2}$ atmosphere. Then, the solid particles are filtered, and washed with deionized water for many times until the residual sulfate and other substances on the surface are cleaned, and finally the solid particles are dried. Eventually, $\mathrm{LiNi}_{0.92} \mathrm{Co}_{0.04} \mathrm{Mn}_{0.04} \mathrm{O}_{2}$ is synthesized by sintering appropriate amount of the precursors and $\mathrm{LiOH}$ at $745{ }^{\circ} \mathrm{C}$ for $15 \mathrm{~h}$ in $\mathrm{O}_{2}$ atmosphere.

Material characterization. The crystal structures of all samples are tested by Powder X-ray diffraction (XRD, Philips Company, $\mathrm{PW}$ 3071, $\mathrm{Cu} \mathrm{K \alpha}$ radiation). The 
morphology and microstructure are observed by scanning electron microscopy (SEM, ZEISS Gemini 300; FIB-SEM, Helios Nanolab 660) and high-resolution transmission electron microscopy (HR-TEM, FEI Strata 400S). The element concentration is detected by Inductively Coupled Plasma Optical Emission Spectrometry (ICP-OES) and energy dispersive spectrometer lines (HAADF-EDS). Malvern laser particle size analyzer is used to analyze secondary particle size distribution. The Nano Measurer is used to count primary particle size.

Flow field simulation and theoretical calculation method. The COMSOL is used to simulate the flow field distribution and calculate the internal stress distribution of particles. In the flow field simulation process, the parameters of reactor and agitator were designed according to the actual size. This model only focuses on the flow condition in the stirred tank and analyzes its influence on the co-precipitation reaction in the tank. Firstly, the wall effect is ignored, and the stirring region model with the same size as the actual size is constructed. The turbulent physical field is added, and the $\mathrm{k}-\varepsilon$ standard model is selected. By using the multiple reference frame method, the internal geometric region is set as the rotating region, and the external geometric region is set as the stationary region. The calculation results of steady flow field can be obtained by using the frozen rotor method. For the stress calculation, the dimensionless particle size, time, Li concentration, and stress are used the definitions previously (The particle size is designed by our actual measurement). ${ }^{35}$ In our calculation, grain density is set as $8960 \mathrm{~kg} \mathrm{~m}^{-3}$, Young's modulus is assumed as 45 GPa for structure, Poisson's ratio is set as 0.27 , grain boundary density is set as 7850 $\mathrm{kg} \mathrm{m}^{-3}$. And a fixed constraint is set. The simulation parameters and the numerical solutions obtained from COMSOL5.5 model are provided. In addition, the anisotropic chemical strain solved. During our charging process, we set the lithium ion density to $80 \%$ in our structure, and during the discharge process, we set the de-lithium ion concentration to $80 \%$ in our systems.

Electrochemical characterization. CR2025 coin-type half-cells are prepared in glove box for testing electrical performances via galvanostatical battery test system utilizing NEWARE software ( $\left.v s . \mathrm{Li} / \mathrm{Li}^{+}, 1 \mathrm{C}=180 \mathrm{mAh} \mathrm{g}^{-1}\right)$ between 2.7 and $4.3 \mathrm{~V}$. 
The electrode plates are prepared with a suitable thick (Active substance loading $3.0 \pm 0.3 \mathrm{mg} \mathrm{cm}^{-2}$ ) by coating acetylene black, binder (PVDF) and active substance on aluminum foil in the weight ratio of 13:7:80. The LiPF6 solution (1.0 M LiPF6 in EC: $\mathrm{DMC}: \mathrm{DEC}=1: 1: 1 \mathrm{vol} \%$ ) and lithium sheet are used for electrolyte and counter electrode, respectively.

\section{Data availability}

The data that support the findings of this study are available from the corresponding author upon reasonable request. 


\section{References}

1. Li W, Erickson EM, Manthiram A. High-nickel layered oxide cathodes for lithium-based automotive batteries. Nature Energy 5, 26-34 (2020)..

2. Qiu L, et al. Polyanion and cation co-doping stabilized Ni-rich Ni-Co-Al material as cathode with enhanced electrochemical performance for Li-ion battery. Nano Energy 63, 103818-103826 (2019).

3. Xiao Y., et al. Deciphering an Abnormal Layered-Tunnel Heterostructure Induced by Chemical Substitution for the Sodium Oxide Cathode. Angew. Chem. Int. Ed. Engl. 59, 1491-1495 (2020).

4. Li J, et al. Structural origin of the high-voltage instability of lithium cobalt oxide. Nat. Nanotechnol. 16,599-605 (2021).

5. Zhu Y. F., et al. Manipulating Layered P2@P3 Integrated Spinel Structure Evolution for High-Performance Sodium-Ion Batteries. Angew. Chem. Int. Ed. Engl. 59, 9299-9304 (2020).

6. Bommel A. van, Dahn J. R. Synthesis of spherical and dense particles of the pure hydroxide phase $\mathrm{Ni}_{1 / 3} \mathrm{Mn}_{1 / 3} \mathrm{Co} 1 / 3(\mathrm{OH})_{2}$. J. Electrochem. Soc. 156, A362-A365 (2009).

7. Bommel A. v., Dahn J. R. Analysis of the Growth Mechanism of Coprecipitated Spherical and Dense Nickel, Manganese, and Cobalt-Containing Hydroxides in the Presence of Aqueous Ammonia. Chem. Mater. 21, 1500-1503 (2009).

8. Yang Y., et al. Growth mechanisms for spherical mixed hydroxide agglomerates prepared by co-precipitation method: A case of $\mathrm{Ni}_{1 / 3} \mathrm{Co}_{1 / 3} \mathrm{Mn}_{1 / 3}(\mathrm{OH})_{2}$. J. Alloys Compd. 619, 846-853 (2015).

9. Yoon C. S., et al. Microstructure Evolution of Concentration Gradient $\mathrm{Li}\left[\mathrm{Ni}_{0.75} \mathrm{Co} 0.10 \mathrm{Mn}_{0.15}\right] \mathrm{O}_{2}$ Cathode for Lithium-Ion Batteries. Adv. Funct. Mater. 28, 1802090-1802096 (2018).

10. Lee M. H., Kang Y. J., Myung S. T., Sun Y. K. Synthetic optimization of $\mathrm{Li}\left[\mathrm{Ni}_{1 / 3} \mathrm{Co}_{1 / 3} \mathrm{Mn}_{1 / 3}\right] \mathrm{O}_{2}$ via co-precipitation. Electrochimi. Acta 50, 939-948 (2004).

11. Shen Y, et al. Insight into the Coprecipitation-Controlled Crystallization Reaction for Preparing Lithium-Layered Oxide Cathodes. ACS Appl. Mater. Interfaces 13, 717-726 (2021). 
12. Qiu L., et al. Exposing microstructure evolution of Ni-Rich Ni-Co-Al hydroxide precursor. Chem. Eng. Sci. 233, 116337-116348 (2021).

13. Yan P, Zheng J, Gu M, Xiao J, Zhang JG, Wang CM. Intragranular cracking as a critical barrier for high-voltage usage of layer-structured cathode for lithium-ion batteries. Nat. Commun. 8, 14101-14109 (2017).

14. Yan $\mathrm{P}$, et al. Coupling of electrochemically triggered thermal and mechanical effects to aggravate failure in a layered cathode. Nat. Commun. 9, 2437-2444 (2018).

15. Kim U-H, et al. Heuristic solution for achieving long-term cycle stability for Ni-rich layered cathodes at full depth of discharge. Nat. Energy 5, 860-869 (2020).

16. Yoon M, et al. Reactive boride infusion stabilizes Ni-rich cathodes for lithium-ion batteries. Nat. Energy 6, 362-371 (2021).

17. Zou L, Li J, Liu Z, Wang G, Manthiram A, Wang C. Lattice doping regulated interfacial reactions in cathode for enhanced cycling stability. Nat. Commun. 10, 3447-3457 (2019).

18. Liu W, et al. Nickel-rich layered lithium transition-metal oxide for high-energy lithium-ion batteries. Angew. Chem. Int. Ed. Engl. 54, 4440-4457 (2015).

19. Wang X, Ding YL, Deng YP, Chen Z. Ni-Rich/Co-Poor Layered Cathode for Automotive Li-Ion Batteries: Promises and Challenges. Adv. Energy Mater. 10, 1903864-1903892 (2020).

20. X. Xu, H. Huo, J. Jian, L. Wang, H. Zhu, S. Xu, X. He, G. Yin, C. Du, X. Sun, Adv. Energy Mater. 9, 1803963-1803971 (2019).

21. Sun YK, et al. Nanostructured high-energy cathode materials for advanced lithium batteries. Nat. Mater. 11, 942-947 (2012).

22. Kim U-H, Lee E-J, Yoon CS, Myung S-T, Sun Y-K. Compositionally Graded Cathode Material with Long-Term Cycling Stability for Electric Vehicles Application. Adv. Energy Mater. 6, 1601417-1601425 (2016).

23. Park K-J, et al. High-Capacity Concentration Gradient $\mathrm{Li}\left[\mathrm{Ni} 0.865 \mathrm{Co} 0.120 \mathrm{Al}_{0.015}\right] \mathrm{O}_{2}$ Cathode for Lithium-Ion Batteries. Adv. Energy Mater. 8, 1703612-1703622 (2018).

24. Ryu H-H, et al. A highly stabilized Ni-rich NCA cathode for high-energy lithium-ion batteries. Mater. Today 36, 73-82 (2020). 
25. Hua W., et al. Shape-controlled synthesis of hierarchically layered lithium transition-metal oxide cathode materials by shear exfoliation in continuous stirred-tank reactors. J. Mater. Chem. A 5, 25391-25402 (2017).

26. Barai P, Feng Z, Kondo H, Srinivasan V. Multiscale Computational Model for Particle Size Evolution during Coprecipitation of Li-Ion Battery Cathode Precursors. J. Phys. Chem. B 123, 3291-3303 (2019).

27. Lv B, Liu Z, Ding R, Wu D, Xu Y. Fast production of $\beta-\mathrm{Ni}(\mathrm{OH})_{2}$ nanostructures with (001) and (100) plane exposure and their electrochemical properties. J. Mater. Chem. A 1, 5695-5699 (2013)..

28. Gao T-P, Wong KW, Ng KM. High-quality LiNi0. ${ }_{8} \mathrm{Co}_{0.15} \mathrm{Al}_{0.05} \mathrm{O}_{2}$ cathode with excellent structural stability: Suppressed structural degradation and pore defects generation. Nano Energy 73, 104798-104807 (2020).

29. Krukau AV, Vydrov OA, Izmaylov AF, Scuseria GE. Influence of the exchange screening parameter on the performance of screened hybrid functionals. J. Chem. Phys. 125, 224106-224111 (2006).

30. P. Harriott. Part II. Suspended in a Pipeline. AIChE J. 8, 101-102 (1962).

31. Liiria M, Koiranenb T, Aittamaaa J. Secondary nucleation due to crystal-impeller and crystal-vessel collisions by population balances in CFD-modelling. J. Cryst. Growth 237-239, 2188-2193 (2002).

32. Bianchini M, Roca-Ayats M, Hartmann P, Brezesinski T, Janek J. There and Back Again-The Journey of $\mathrm{LiNiO}_{2}$ as a Cathode Active Material. Angew. Chem. Int. Ed. Engl 58, 10434-10458 (2019).

33. Zou L, et al. The Role of Secondary Particle Structures in Surface Phase Transitions of Ni-Rich Cathodes. Chem. Mater. 32, 2284-2892 (2020).

34. Ulvestad A., et al. Topological defect dynamics in operando battery nanoparticles. Science 348, 1344-1347 (2015).

35. Bi Y., et al. Reversible planar gliding and microcracking in a single-crystalline Ni-rich cathode. Scienc. 370, 1313-1317 (2020).

\section{Acknowledgments}

This work was supported by the project from the National Natural Science Foundation 
of China [20A20145, 21878195, 21805198], the Distinguished Young Foundation of Sichuan Province [2020JDJQ0027], 2020 Strategic cooperation project between Sichuan University and Zigong Municipal People's Government [No. 2020CDZG-09], State Key Laboratory of Polymer Materials Engineering [No. sklpme2020-3-02], Sichuan Provincial Department of Science and Technology [No. 2020YFG0471, No. 2020YFG0022], Sichuan Province Science and Technology Achievement Transfer and Trans-formation Project [No 21ZHSF0111]. Sichuan University postdoctoral interdisciplinary Innovation Fund [2021SCU12084].

\section{Author contributions}

Lang Qiu: Formal analysis, Conceptualization, Data curation, Writing - original draft. Mengke Zhang: Formal analysis, Data curation, Writing - original draft. Kanghui Hu: Formal analysis, Data curation. Yang Song: Writing - original draft, Formal analysis, Data curation. Zhenguo Wu: Formal analysis, Data curation. Luchao Yue: Formal analysis, Data curation. Wei Xiang: Formal analysis, Data curation. Yong Ming: Formal analysis, Data curation. Yuxia Liu: Formal analysis, Data curation. Gongke Wang: Formal analysis, Data curation. Yan Sun: Formal analysis, Data curation. Jun

Zhang: Formal analysis, Data curation. Bin Zhang: Formal analysis, Data curation. Xiaodong Guo: Writing - original draft, Formal analysis, Data curation.

\section{Competing interests}

The authors declare no competing interests.

\section{Additional information}

Supporting Information is available from the author. 


\section{Supplementary Files}

This is a list of supplementary files associated with this preprint. Click to download.

- Surpporting.docx 